\title{
Erratum
}

\section{Erratum to "Detection of Alternative Drugs for Illegal Injection Based on Surface-Enhanced Raman Spectroscopy"}

\author{
Lin Bao $\mathbb{D}^{1,2}$ Siqingaowa Han $\mathbb{D}^{3},{ }^{3}$ Xuanyu Sha $\mathbb{D}^{1},{ }^{1}$ Hang Zhao $\mathbb{D}^{1},{ }^{1}$ Yuping Liu $\left(\mathbb{D},{ }^{1}\right.$ \\ Dianyang Lin $\mathbb{D D}^{1}$, and Wuliji Hasi $\mathbb{D}^{1}$ \\ ${ }^{1}$ National Key Laboratory of Science and Technology on Tunable Laser, Harbin Institute of Technology, Harbin 150080, China \\ ${ }^{2}$ College of Physics and Electronics Information, Inner Mongolia University for Nationalities, Tongliao 028043, \\ Inner Mongolia, China \\ ${ }^{3}$ Affiliated Hospital, Inner Mongolia University for the Nationalities, Tongliao 028007, Inner Mongolia, China
}

Correspondence should be addressed to Siqingaowa Han; hansiqin@126.com and Wuliji Hasi; hasiwuliji@126.com

Received 28 June 2020; Accepted 28 June 2020; Published 13 February 2021

Copyright (C) 2021 Lin Bao et al. This is an open access article distributed under the Creative Commons Attribution License, which permits unrestricted use, distribution, and reproduction in any medium, provided the original work is properly cited.

In the article titled "Detection of Alternative Drugs for Illegal Injection Based on Surface-Enhanced Raman Spectroscopy" [1], there was an error in the corresponding author information which was published as "Siqingaowa Han, Yuping Liu, and Dianyang Lin." This error was introduced during the production of the article and Hindawi apologises for this error. The correct corresponding authors are "Siqingaowa Han and Wuliji Hasi."

The corrected corresponding authors are shown in the corresponding author line above.

\section{References}

[1] B. Lin, H. Siqingaowa, S. Xuanyu et al., "Detection of Alternative Drugs for Illegal Injection Based on Surface-Enhanced Raman Spectroscopy," Journal of Spectroscopy, vol. 2019, pp. 1-5, Article ID 7458371, 2019. 